\title{
IDENTIFICAÇÃO DOS RELACIONAMENTOS ENTRE OS FATORES CRÍTICOS DE SUCESSO, BARREIRAS E PRÁTICAS PARA A IMPLEMENTAÇÃO ENXUTA EM UMA PEQUENA EMPRESA
}

\section{IDENTIFICATION OF RELATIONSHIPS BETWEEN THE CRITICAL SUCCESS FACTORS, BARRIERS AND PRACTICES FOR IMPLEMENTATION OF LEAN PRODUCTION ON A SMALL COMPANY}

\author{
Laís do Nascimento Ghizoni Pereira* E-mail: laisghizoni@hotmail.com \\ Guilherme Luz Tortorella* E-mail: gluztortorella@gmail.com \\ *Universidade Federal de Santa Catarina (UFSC), Florianópolis, SC
}

\begin{abstract}
Resumo: A adoção dos princípios e práticas oriundos da Produção Enxuta (PE) auxilia na eliminação sistemática dos desperdícios, ao passo que lapida a cultura organizacional para a sustentação da melhoria contínua. O processo de implementação da PE pode variar de acordo com os problemas existentes e o contexto no qual a empresa está inserida. Assim, este artigo busca identificar como se relacionam os fatores críticos de sucesso, barreiras e práticas para a implementação enxuta em uma pequena empresa. Para isso, os principais FCS, barreiras e práticas de PE relacionados a pequenas empresas foram inicialmente consolidados a partir de uma revisão bibliográfica. Através de entrevistas semiestruturadas e grupos focados com as lideranças de uma pequena empresa manufatureira do setor eletrônico em implementação enxuta, as relações entre FCS, barreiras e práticas enxutas foram avaliadas e ranqueadas a partir da incorporação de uma ferramenta de análise multicriterial. Além da contribuição teórica já evidenciada, este estudo apresenta implicações de natureza prática/gerencial, uma vez que provê um direcionamento para auxiliar a implementação enxuta em pequenas empresas manufatureiras. Adicionalmente, o maior entendimento dessas relações sob o contexto em estudo possibilita aos gestores a antecipação de potencias problemas, permitindo uma implementação enxuta mais bem-sucedida. Ao final os resultados foram analisados e concluídos com sugestões para futuras pesquisas.
\end{abstract}

Palavras-chave: Produção Enxuta.Pequenas empresas. Fatores críticos de sucesso. Práticas enxutas. AHP.

\begin{abstract}
The Lean Production implementation process can vary according to the existing problems and the context in which the company is inserted. Thus, this article aims to identify how the critical success factors, barriers and practices for lean implementation in a small company. For this, the main FCS, barriers and practices of PE related to small companies were initially consolidated from a bibliographic review. Through semi-structured interviews and focus groups with the leaders of a small electronics manufacturing company in lean implementation, the relationships between FCS, barriers and lean practices were evaluated and ranked through the incorporation of a multi-criteria analysis tool. In addition to the theoretical contribution already made, this study presentes practical implications, since it provides a guide to assist the lean implementation in small manufacturing companies. In addition, the greater understanding of these relationships under the context under study enables managers to anticipate potential problems, allowing a more successful lean implementation. At the end the results were analyzed and concluded with suggestions for future research.
\end{abstract}

Keywords: Lean Production. Small Company. Critical Success Factors. Lean Practices. AHP. 


\section{INTRODUÇÃO}

A adoção dos princípios e práticas oriundos da Produção Enxuta (PE) auxilia na eliminação sistemática dos desperdícios, ao passo que lapida a cultura organizacional para a sustentação da melhoria contínua (WOMACK et al., 1992). O processo de implementação da PE pode variar de acordo com os problemas existentes e o contexto no qual a empresa está inserida (MARODIN et al., 2015). Dentre as variáveis contextuais, o porte da empresa, geralmente associado ao número de funcionários, é enfatizado como um dos fatores mais relevantes a ser considerado, visto que influencia diversos aspectos chaves da implementação enxuta, tais como disponibilidade de recursos, estruturação e padronização de processos, níveis hierárquicos, etc. (SHAH; WARD, 2003; ACHANGA et al.,2006; NORDIN et al., 2010).

Especificamente no contexto de pequenas empresas, estudos anteriores (SAURIN et al., 2010; ABOLHASSANI et al., 2016) apontam dificuldades no processo de implementação da PE que diferem de empresas de maior porte. Assim, cabe destacar determinados fatores críticos de sucesso (FCS) e barreiras para o processo de implementação da PE nas empresas de pequeno porte, tais como a proximidade entre a alta gerência (geralmente sendo representada pelos proprietários da pequena empresa) e a operação, o que tende a favorecer maior suporte aos projetos de melhoria (ANTONY et al., 2005; HALLGREN; OLHAGER, 2009; ANAND; KODALI, 2010).

Soma-se a isso o fato de haver uma crescente quantidade de empresas de pequeno porte, aumentando sua representatividade socioeconômica (ANTONY et al., 2005). Na Inglaterra, por exemplo, as pequenas empresas (caracterizadas por empregarem até 100 funcionários) geram pelo menos $50 \%$ dos empregos no país (ACHANGA et al., 2006). Tal importância é observada também no Brasil, onde as empresas de menor porte, as quais empregam de 50 a 100 funcionários, representam um quarto do PIB (Produto Interno Bruto) (SEBRAE, 2014). Além disso, as pequenas empresas compreendem também $99 \%$ dos estabelecimentos formais $e$ empregam 52\% da mão de obra no Brasil (NOGUEIRA; OLIVEIRA, 2013).

Assim, apesar de haver estudos investigando o efeito do porte da empresa sobre o nível de adoção da PE, estes abordam esta problemática de forma superficial ou tangencial, sem explorar as relações entre FCS, barreiras e práticas 
de um modo mais holístico (DOMBROWSKI et al., 2010; PEREIRA; TORTORELLA, 2016). Além disso, os frameworks existentes para implementação enxuta são direcionados para empresas de maior porte, desconsiderando o contexto específico de pequenas empresas (ANAND; KODALI, 2010; BELHADI; TOURIKI, 2016). A partir do exposto anteriormente, pode-se formular a seguinte questão de pesquisa: "Quais são as relações entre os fatores críticos de sucesso, barreiras e práticas para a implementação de produção enxuta em uma pequena empresa? ".

Assim, este artigo busca identificar como se relacionam os fatores críticos de sucesso, barreiras e práticas para a implementação enxuta em uma pequena empresa. Para isso, os principais FCS, barreiras e práticas de PE relacionados a pequenas empresas foram inicialmente consolidados a partir de uma revisão bibliográfica. Através de entrevistas semiestruturadas e grupos focados com as lideranças de uma pequena empresa manufatureira do setor eletrônico em implementação enxuta, as relações entre FCS, barreiras e práticas enxutas foram avaliadas e ranqueadas a partir da incorporação de uma ferramenta de análise multicriterial. Além da contribuição teórica já evidenciada, este estudo apresenta implicações de natureza prática/gerencial, uma vez que provê um direcionamento para auxiliar a implementação enxuta em pequenas empresas manufatureiras. Adicionalmente, o maior entendimento dessas relações sob o contexto em estudo possibilita aos gestores a antecipação de potencias problemas, permitindo uma implementação enxuta mais bem-sucedida.

Além desta seção introdutória, o presente artigo apresenta na seção 2 uma revisão da literatura a respeito dos FCS, barreiras e práticas de implementação da PE em pequenas empresas. A seção 3 descreve o método proposto, cujos resultados de sua aplicação estão explanados na seção 4. Por fim, a seção 5 encerra 0 trabalho apresentando as conclusões e oportunidades para futuros trabalhos.

\section{REVISÃO BIBLIOGRÁFICA}

Segundo Womack et al. (1992) a PE busca a eliminação de desperdícios, maximização da eficiência, produtividade e flexibilidade. Apesar de a PE apresentar evidências de benefícios em diversos segmentos, ainda há desafios a serem superados no seu processo de implementação. Tais desafios podem prejudicar Revista Produção Online. Florianópolis, SC, v. 18, n. 4, p. 1422-1444, 2018. 
significativamente o sucesso da PE, em especial no caso de pequenas empresas (ACHANGA et al., 2006). Geralmente, essas empresas carecem de lideranças capacitadas que disseminem a cultura da melhoria contínua. Nesse sentido torna-se importante entender os fatores críticos que corroboram para a implementação enxuta nesse contexto (BAKAS et al., 2011; GODINHO FILHO et al., 2016).

Tabela 1 - FCS e barreiras para implementação da PE identificados na literatura

\begin{tabular}{|c|c|c|c|c|c|c|c|c|c|c|c|c|c|c|c|c|c|}
\hline \multirow{2}{*}{ Artigos } & \multicolumn{13}{|c|}{ FCS } & \multicolumn{4}{|c|}{ Barreiras } \\
\hline & $f_{1}$ & $f_{2}$ & $f_{3}$ & $f_{4}$ & $f_{5}$ & $f_{6}$ & $f_{7}$ & $f_{8}$ & $f_{9}$ & $f_{10}$ & $f_{11}$ & $f_{12}$ & $f_{13}$ & $b_{1}$ & $b_{2}$ & $b_{3}$ & $b_{4}$ \\
\hline BAKAS et al. (2011) & $\mathrm{X}$ & $\mathrm{X}$ & & & $\mathrm{X}$ & & $\mathrm{X}$ & $\mathrm{X}$ & $x$ & $\mathrm{x}$ & $\mathrm{X}$ & $x$ & & $x$ & $\mathrm{X}$ & $\mathrm{X}$ & \\
\hline KUMAR et al. (2014) & $x$ & $x$ & $\mathrm{x}$ & $\mathrm{x}$ & $x$ & & $x$ & $x$ & & & $x$ & & $\mathrm{x}$ & $x$ & $x$ & & \\
\hline $\begin{array}{l}\text { KUMAR; ANTONY } \\
\text { (2009) }\end{array}$ & $x$ & $x$ & $x$ & $x$ & $\mathrm{x}$ & $\mathrm{x}$ & $\mathrm{x}$ & $x$ & & & & $x$ & & & & $\mathrm{x}$ & \\
\hline KUMAR et al. (2009) & $x$ & $\mathrm{x}$ & $\mathrm{x}$ & $\mathrm{X}$ & $\mathrm{x}$ & & $\mathrm{X}$ & $\mathrm{x}$ & & & $\mathrm{x}$ & & $\mathrm{x}$ & & $\mathrm{x}$ & & \\
\hline $\begin{array}{l}\text { GODINHO FILHO et al. } \\
(2016)\end{array}$ & $x$ & $\mathrm{x}$ & $\mathrm{x}$ & $\mathrm{x}$ & & $\mathrm{x}$ & $\mathrm{x}$ & $\mathrm{x}$ & $\mathrm{x}$ & & & & & $\mathrm{x}$ & & & \\
\hline $\begin{array}{l}\text { WORLEY; DOOLEN } \\
(2006)\end{array}$ & $x$ & & & & $\mathrm{x}$ & $\mathrm{x}$ & $\mathrm{x}$ & $x$ & $\mathrm{x}$ & & $\mathrm{x}$ & $\mathrm{x}$ & & & & & \\
\hline TIMANS et al. (2012) & & $\mathrm{x}$ & & $x$ & $\mathrm{x}$ & $\mathrm{x}$ & $\mathrm{x}$ & $\mathrm{x}$ & & $x$ & & & $\mathrm{x}$ & & & & \\
\hline SAURIN et al. (2010) & & $\mathrm{x}$ & & & & & $\mathrm{x}$ & & & $\mathrm{x}$ & & & & $\mathrm{x}$ & $\mathrm{x}$ & $\mathrm{x}$ & $\mathrm{X}$ \\
\hline $\begin{array}{l}\text { BELHADI; TOURIKI } \\
(2016)\end{array}$ & $\mathrm{x}$ & $\mathrm{x}$ & & $x$ & $\mathrm{x}$ & $\mathrm{x}$ & & & $\mathrm{x}$ & & & $\mathrm{x}$ & & & & & \\
\hline $\begin{array}{l}\text { DOMBROWSKI; } \\
\text { MIELKE (2014) }\end{array}$ & $x$ & $x$ & $x$ & $x$ & & & & $\mathrm{x}$ & & $\mathrm{x}$ & & $x$ & & & & & \\
\hline ANTONY et al. (2005) & $x$ & & $\mathrm{x}$ & $\mathrm{x}$ & $\mathrm{x}$ & $\mathrm{x}$ & & $\mathrm{x}$ & $x$ & & & & & & & & \\
\hline NORDIN et al. (2012) & $\mathrm{x}$ & $\mathrm{x}$ & $\mathrm{x}$ & & $\mathrm{x}$ & & $\mathrm{x}$ & & & $\mathrm{x}$ & & & & $\mathrm{x}$ & & & \\
\hline $\begin{array}{l}\text { BHAMU; SANGWAN } \\
\text { (2014) }\end{array}$ & $x$ & $x$ & $\mathrm{x}$ & & & & & & $x$ & & & $x$ & & $\mathrm{x}$ & & & \\
\hline ANAND; KODALI (2010) & $\mathrm{x}$ & $\mathrm{x}$ & $\mathrm{x}$ & & & $\mathrm{x}$ & & & & & $\mathrm{x}$ & & & & $x$ & & \\
\hline NETLAND (2016) & $\mathrm{x}$ & & & & $\mathrm{x}$ & & & & $\mathrm{x}$ & $\mathrm{x}$ & $\mathrm{x}$ & $\mathrm{x}$ & & & & & \\
\hline ACHANGA et al. (2006) & $x$ & $\mathrm{x}$ & $\mathrm{x}$ & & & $\mathrm{x}$ & & & $x$ & & & & & & & & \\
\hline BHASIN (2012) & & & $\mathrm{x}$ & & & & & $\mathrm{x}$ & & & $\mathrm{x}$ & & & $\mathrm{x}$ & $\mathrm{x}$ & & \\
\hline MANVILLE et al. (2012) & & & $x$ & $\mathrm{x}$ & $\mathrm{x}$ & $\mathrm{x}$ & & & & & $x$ & & & & & & \\
\hline ZHOU (2016) & & & & & & & & & & & & & & $\mathrm{x}$ & $x$ & & $\mathrm{x}$ \\
\hline $\begin{array}{l}\text { DOMBROWSKI et al. } \\
\text { (2010) }\end{array}$ & $\mathrm{x}$ & & & $x$ & & & $\mathrm{x}$ & & & & & & & & & & \\
\hline MATT; RAUCH (2013) & & & $\mathrm{x}$ & & & & & & $x$ & & & & & & & & \\
\hline $\begin{array}{l}\text { ABOLHASSANI et al. } \\
\text { (2016) }\end{array}$ & & & & & & & & & & & & & & $\mathrm{x}$ & $x$ & & \\
\hline NORDIN et al. (2010) & & & & & & & & & & & & & & $\mathrm{x}$ & $x$ & & \\
\hline JADHAV et al. (2014) & & & & & & & & & & & & & & $x$ & $x$ & & \\
\hline WONG; WONG (2009) & & $\mathrm{x}$ & & & & & & & & & & & & & & & \\
\hline SIM; ROGERS (2008) & & & & & & & & & & & & & & $\mathrm{x}$ & & & \\
\hline Total & 15 & 14 & 13 & 10 & 11 & 9 & 10 & 10 & 9 & 6 & 8 & 7 & 3 & 11 & 10 & 3 & 2 \\
\hline
\end{tabular}

FCS: $f_{1}$ - Liderança; $f_{2}$ - Cultura; $f_{3}$ - Competências/ Habilidades; $f_{4}$ - Alinhamento à estratégia em todos os níveis; $f_{5}$ - Educação e treinamento; $f_{6}$ - Compromisso da alta administração; $f_{7}$ - Comunicação (metas e objetivos com iniciativas de melhoria); $f_{8}$ - Envolvimento com os stakeholders; $f_{9}$ - Alocação de recursos; $f_{10}$ - Aprendizagem contínua; $f_{11}$ - Comprometimento/ Motivação dos trabalhadores; $f_{12}$ - Avaliação de desempenho (foco em longo prazo); $f_{13}$ - Infraestrutura Organizacional.

Barreiras: $b_{1}$ - Falta de compreensão dos benefícios; $b_{2}$ - Resistência à mudança pelos funcionários; $b_{3}$ Dificuldade de adaptar conceitos e práticas; $b_{4}$ - Falha de projetos de melhoria anteriores.

Fonte: Adaptado de Pereira e Tortorella (2016) 
Além dos FCS, é importante compreender também alguns obstáculos que podem vir a impedir a implementação da PE (YANG; YUJU, 2010; ZHOU, 2016). De uma maneira geral, uma barreira para a implementação da PE pode ser configurada como a pouca ênfase a um determinado FCS (ANAND; KODALI, 2010). Nesse sentido, a Tabela 1 consolida os principais FCS e barreiras encontrados na literatura voltados para o contexto da implementação da PE em pequenas empresas.

Além disso cabe destacar algumas práticas enxutas, de modo que tais práticas apresentam aplicação e diferentes intensidades de adoção dependendo do contexto da empresa, necessidade de investimento ou problemas existentes (MATT, 2008; KUMAR et al., 2014). Abolhassani et al. (2016) alegam que empresas de maior porte têm a tendência de adotarem mais amplamente as práticas enxutas. Portanto, a identificação das práticas mais facilmente aplicáveis ao contexto das pequenas empresas, bem como seu método de implementação são pontos importantes para a mudança enxuta (SHAH; WARD, 2003).

Logo, a implementação de algumas práticas enxutas pode requerer maior investimento de recursos do que outras, o que eventualmente dificulta sua aplicação em empresas de pequeno porte cuja disponibilidade de recursos é mais restrita. Analogamente aos FCS e barreiras, a Tabela 2 apresenta as principais práticas enxutas aplicáveis às pequenas empresas identificadas a partir de pesquisas evidenciadas na literatura. Apesar da desigualdade de frequência de citação, verifica-se a existência de 19 práticas fundamentais para a implementação da PE em pequenas empresas. 
Tabela 2 - Principais práticas enxutas aplicadas em pequenas empresas

\begin{tabular}{|c|c|c|c|c|c|c|c|c|c|c|c|c|c|c|c|c|c|c|c|}
\hline \multirow{2}{*}{ Artigos } & \multicolumn{19}{|c|}{ Práticas enxutas } \\
\hline & $p_{1}$ & $p_{2}$ & $p_{3}$ & $p_{4}$ & $p_{5}$ & $p_{6}$ & $p_{7}$ & $p_{8}$ & $p_{9}$ & $p_{10}$ & $p_{11}$ & $p_{12}$ & $p_{13}$ & $p_{14}$ & $p_{15}$ & $p_{16}$ & $p_{17}$ & $p_{18}$ & $p_{19}$ \\
\hline ZHOU (2016) & $x$ & $x$ & $x$ & $\mathrm{x}$ & $x$ & & & $x$ & $\mathrm{x}$ & & $x$ & $\mathrm{x}$ & $\mathrm{X}$ & & & $x$ & $\mathrm{X}$ & $\mathrm{X}$ & $\mathrm{X}$ \\
\hline SHAH; WARD (2003) & $\mathrm{x}$ & $\mathrm{X}$ & $\mathrm{x}$ & & $\mathrm{X}$ & $\mathrm{X}$ & $x$ & $\mathrm{X}$ & $\mathrm{x}$ & $\mathrm{x}$ & & & & $\mathrm{X}$ & & & $\mathrm{X}$ & & \\
\hline $\begin{array}{l}\text { DOOLEN; HACKER } \\
(2005)\end{array}$ & & $\mathrm{X}$ & & $\mathrm{X}$ & $\mathrm{x}$ & $\mathrm{X}$ & $\mathrm{x}$ & $\mathrm{x}$ & $\mathrm{x}$ & $\mathrm{X}$ & $\mathrm{x}$ & $\mathrm{x}$ & & & & & & & \\
\hline ROSE et al. (2011) & $\mathrm{X}$ & $\mathrm{X}$ & $\mathrm{X}$ & $\mathrm{X}$ & & $\mathrm{X}$ & $X$ & & & $\mathrm{X}$ & & $\mathrm{X}$ & & $\mathrm{X}$ & & $\mathrm{X}$ & & & \\
\hline ROSE et al. (2013) & & $\mathrm{X}$ & $\mathrm{x}$ & $\mathrm{x}$ & $\mathrm{X}$ & $\mathrm{X}$ & & $\mathrm{x}$ & & & & $\mathrm{x}$ & & $\mathrm{X}$ & & & & $\mathrm{X}$ & $\mathrm{x}$ \\
\hline SAURIN et al. (2010) & $\mathrm{X}$ & $\mathrm{X}$ & $\mathrm{x}$ & & & & $\mathrm{x}$ & & $\mathrm{x}$ & & $\mathrm{x}$ & $\mathrm{X}$ & & $\mathrm{X}$ & & $\mathrm{X}$ & & & \\
\hline MATT; RAUCH (2013) & $\mathrm{x}$ & & $\mathrm{x}$ & $\mathrm{x}$ & $\mathrm{x}$ & $\mathrm{x}$ & & $\mathrm{X}$ & $\mathrm{x}$ & & & & & & & $\mathrm{x}$ & & $\mathrm{x}$ & \\
\hline KUMAR et al. (2006) & $\mathrm{x}$ & $\mathrm{X}$ & $\mathrm{x}$ & $\mathrm{X}$ & $x$ & $\mathrm{x}$ & & $\mathrm{X}$ & & & $\mathrm{x}$ & & $\mathrm{X}$ & & & & & & \\
\hline $\begin{array}{l}\text { BELHADI; TOURIKI } \\
\text { (2016) }\end{array}$ & $x$ & $\mathrm{X}$ & $\mathrm{x}$ & $\mathrm{X}$ & & & & $\mathrm{x}$ & & & $\mathrm{x}$ & & $\mathrm{x}$ & & $\mathrm{x}$ & & & & \\
\hline $\begin{array}{l}\text { BHAMU; SANGWAN } \\
\text { (2014) }\end{array}$ & $x$ & $\mathrm{x}$ & & $\mathrm{x}$ & $\mathrm{x}$ & & $x$ & $\mathrm{x}$ & $\mathrm{x}$ & & $\mathrm{x}$ & & & & & & & & \\
\hline KUMAR et al. (2014) & $\mathrm{X}$ & $\mathrm{x}$ & $\mathrm{x}$ & & $\mathrm{X}$ & & $x$ & $\mathrm{X}$ & & & & & $\mathrm{x}$ & & & & & & \\
\hline WONG; WONG (2009) & $x$ & $x$ & $\mathrm{x}$ & $\mathrm{x}$ & $\mathrm{x}$ & & & & & & & $\mathrm{x}$ & & & & & & & $\mathrm{x}$ \\
\hline $\begin{array}{l}\text { GODINHO FILHO et al. } \\
\text { (2016) }\end{array}$ & $\mathrm{x}$ & $\mathrm{X}$ & & & $\mathrm{x}$ & $\mathrm{x}$ & & & & & & & & & $\mathrm{x}$ & & $\mathrm{x}$ & & \\
\hline $\begin{array}{l}\text { ABOLHASSANI et al. } \\
\text { (2016) }\end{array}$ & $\mathrm{X}$ & $\mathrm{x}$ & & & & $\mathrm{X}$ & & & $\mathrm{x}$ & & & & & & & & $\mathrm{X}$ & $\mathrm{x}$ & \\
\hline SHAH; WARD (2007) & $\mathrm{x}$ & $x$ & & & $\mathrm{x}$ & $\mathrm{x}$ & & & & & & & & & $\mathrm{x}$ & & $\mathrm{x}$ & & \\
\hline $\begin{array}{l}\text { WORLEY; DOOLEN } \\
\text { (2006) }\end{array}$ & $\mathrm{x}$ & & $\mathrm{x}$ & $\mathrm{x}$ & & & & & $\mathrm{x}$ & & $\mathrm{x}$ & & & & & & & & \\
\hline KUMAR; ANTONY (2009) & & & & $\mathrm{x}$ & & $\mathrm{x}$ & $\mathrm{x}$ & & & & & & $\mathrm{x}$ & & & & & & \\
\hline KUMAR et al. (2009) & & & $\mathrm{X}$ & & & & $\mathrm{x}$ & & & $\mathrm{X}$ & & & $\mathrm{X}$ & & & & & & \\
\hline $\begin{array}{l}\text { DOMBROWSKI; MIELKE } \\
\text { (2014) } \\
\text { SANCHEZ; PEREZ } \\
(2001)\end{array}$ & $\mathrm{x}$ & & $\mathrm{x}$ & $x$ & $\mathrm{X}$ & & & & $\mathrm{x}$ & $\mathrm{x}$ & & & & $\mathrm{x}$ & & & & & $x$ \\
\hline ANTONY et al. (2005) & & & & & & & $\mathrm{X}$ & & & & & & & & $\mathrm{X}$ & & & $\mathrm{x}$ & \\
\hline ANAND; KODALI (2010) & & & $\mathrm{x}$ & & & & & & & $\mathrm{x}$ & & & & & & $\mathrm{x}$ & & & \\
\hline $\begin{array}{l}\text { DOMBROWSKI et al. } \\
\text { (2010) } \\
\text { HALLGREN; OLHAGER } \\
\text { (2009) }\end{array}$ & & $\mathrm{x}$ & $\mathrm{x}$ & $x$ & & & $\mathrm{X}$ & & & & & $\mathrm{x}$ & & & $\mathrm{x}$ & & & & \\
\hline $\begin{array}{l}\text { DORA et al. (2014) } \\
\text { TORTORELLA et al. } \\
\text { (2015) }\end{array}$ & $\mathrm{x}$ & $x$ & & & & & & & & $\mathrm{x}$ & & & & $\mathrm{x}$ & & & & & \\
\hline Total & 16 & 16 & 15 & 13 & 12 & 10 & 10 & 9 & 9 & 7 & 7 & 7 & 6 & 6 & 5 & 5 & 5 & 5 & 4 \\
\hline
\end{tabular}

Práticas: $p_{1}$ - Produção puxada; $p_{2}$ - Manutenção produtiva total; $p_{3}$ - Kaizen / Grupos de melhoria continua; $p_{4}$ - 5S; $p_{5}$ - Just in time (JIT); $p_{6}$ - Redução de tempo de ciclo; $p_{7}$ - Gestão da Qualidade Total; $p_{8}$ - Produção celular; $p_{9}$ - Troca rápida de ferramentas; $p_{10}$ - Envolvimento das pessoas; $p_{11}$ Mapeamento de Fluxo de Valor (MFV); $p_{12}$ - Padronização do trabalho; $p_{13}$ - Lean seis sigma; $p_{14}$ Equipe multifuncional; $p_{15}$ - Controle Estatístico de Processos (SPC); $p_{16}$ - Gerenciamento visual; $p_{17}$ Fluxo contínuo; p18 - Poka Yoke; p19 - Plan, do, check, act (PDCA).

Fonte: Adaptado de Pereira e Tortorella (2016)

De um modo geral, as Tabelas 1 e 2 destacam as principais variáveis a serem consideradas por pequenas empresas que adotam o paradigma enxuto. Contudo, cabe destacar que são poucos os estudos que relacionam efetivamente as 3 variáveis (FCS, barreiras e práticas) concomitantemente. Tal lacuna evidencia a Revista Produção Online. Florianópolis, SC, v. 18, n. 4, p. 1422-1444, 2018. 
necessidade de estudos mais holísticos que permitam o entendimento sistêmico da implementação enxuta.

\section{MÉTODO PROPOSTO}

O método proposto para este trabalho é compreendido por seis etapas: ( $I$ ) definição dos participantes dos grupos focados; (ii) estruturação das hipóteses e roteiro de discussão; (iii) realização dos encontros de grupos focados; (iv) realização de observação participativa na empresa; $(v)$ análise e tratamento dos dados coletados; $(v i)$ análise multicriterial entre práticas enxutas, barreiras e FCS; e (vii) ranqueamento dos FCS e barreiras para favorecer a implementação enxuta.

A etapa (i) trata da definição dos participantes para a realização do método de grupos focados, os quais visam capturar diferentes pontos de vistas no estabelecimento de um senso comum sobre um determinado tema (RIBEIRO; NEWMANN, 2012). A técnica de grupos focados tem sido usada para coleta de dados em ambiente no qual as informações não estão bem estruturadas (TORTORELLA, 2008), de maneira que a escolha das variáveis influenciadoras no processo pode ser determinada pela percepção dos indivíduos. Os participantes dos grupos são escolhidos de acordo com o propósito da pesquisa; neste caso, são formados dois grupos: (I) um com membros estratégicos da empresa (gerentes e coordenadores das áreas) e (ii) outro composto por líderes operacionais (supervisores de produção). Além disso, é desejado que os participantes de ambos os grupos possuam algumas características complementares, tais como: conhecimento da operação e do ambiente de negócio da empresa, conhecimento geral dos setores, facilidade de expressão e clareza na formulação de conceitos.

A etapa (ii) consiste em, baseado nas práticas, FCS e barreiras identificadas nas Tabelas 1 e 2, elencar quais são pertinentes na empresa em estudo, de tal forma a estabelecer posteriormente o relacionamento entre estes. Para tal, estrutura-se nesta etapa as hipóteses e roteiro para discussões nos grupos focados. Ribeiro e Newmann (2012) sugerem que o roteiro das discussões abordem cinco tipos principais de perguntas: (a) questão inicial, (b) questão de transição, (c) questões centrais, ( $d$ ) questão de resumo, e (e) questão final. A questão inicial tem como objetivo tranquilizar e descontrair o ambiente, permitindo que os participantes envolvidos se sintam à vontade para a discussão. Já a questão de transição encaminha a discussão para o foco do problema, de modo que as questões centrais possam definitivamente abordar o interesse de pesquisa e capturar a informação almejada. Na sequência, busca-se sintetizar o que foi debatido, através da questão Revista Produção Online. Florianópolis, SC, v. 18, n. 4, p. 1422-1444, 2018. 
de resumo, de forma a consolidar os principais pontos e direcionar para a conclusão da discussão a partir da questão final. A Figura 1 apresenta o roteiro criado para a realização dos encontros com os grupos focados, sendo que ao lado de cada pergunta apresenta-se o tipo da mesma de acordo com a sugestão de Ribeiro e Newmann (2012). As perguntas 5 e 6 do roteiro questionam a repeito das listas de práticas e de FCS/barreiras identificadas na literatura para pequenas empresas. Portanto, neste momento, o moderador apresenta as Tabelas 1 e 2 para discussão em grupo. Salienta-se que o conteúdo das perguntas do roteiro não é de conhecimento dos participantes antes dos encontros, o que contribui para que, de certo modo, apareçam as diferenças de percepção entre membros do grupo quanto a aspectos relevantes da pesquisa. Além disso, esta etapa aborda o planejamento dos grupos focados, incluindo a definição do local, data e hora dos encontros.

A etapa (iii) consiste efetivamente na realização dos encontros dos grupos focados. Cabe destacar que antes de seu início os participantes são questionados se há algum empecílio para gravar o encontro; em havendo, as informações são registradas apenas através de anotações sobre os comentários realizados. Em seguida, o moderador explica as regras básicas da sessão, incluindo tempo da mesma, o caráter sigiloso e anônimo das informações compartilhadas e a forma dos participantes responderem às questões. Por exemplo, o moderador pode estabelecer que cada membro, ao redor da mesa, responda cada questão, ordenadamente, na sua vez (RIBEIRO, 2000).

Figura 1 - Roteiro para os encontros de grupos focados

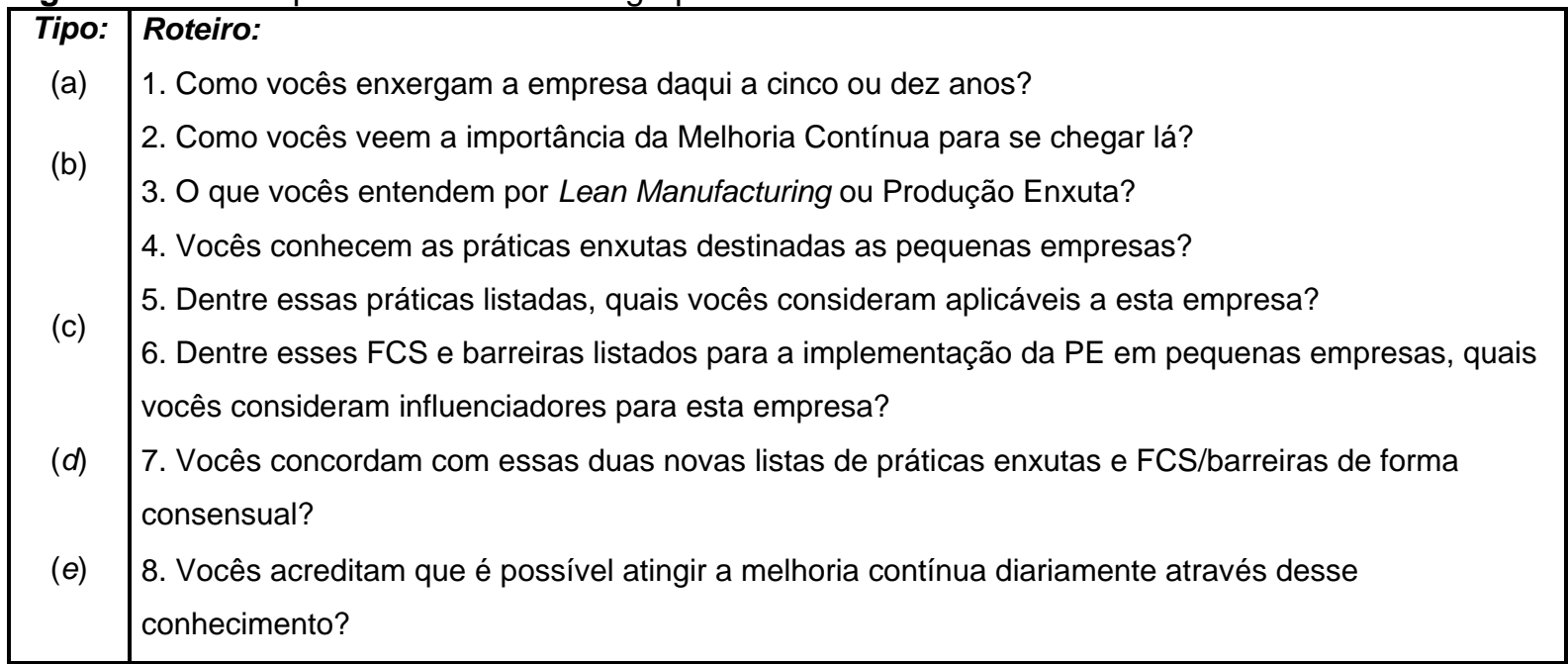

$\mathrm{Na}$ etapa (iv), tem-se a realização de observação participativa na empresa, a Revista Produção Online. Florianópolis, SC, v. 18, n. 4, p. 1422-1444, 2018. 
qual consiste na coleta de informações complementares a partir da visão pessoal do pesquisador no ambiente em estudo (RIBEIRO, 2003). Essa observação possibilita a coleta de dados de interesse do pesquisador, fornecendo-lhe subsídios para maior entendimento e compreensão das informações obtidas a partir da etapa (iii), ou mesmo para estabelecer referenciais que permitem avançar na pesquisa. Analogamente à (iii), esta etapa deve seguir uma sistemática estruturada para assegurar que a informação obtida seja confiável e válida (BEYEA; NICOLL, 2000). Nesse sentido, com o intuito de complementar quais as práticas, FCS e barreiras são pertinentes à pequena empresa em estudo, alguns critérios foram estabelecidos para a observação participativa, tais como: (a) a acompanhar as etapas produtivas do processo para uma maior familiaridade com a rotina da empresa; (b) a verificação das limitações de processo/operação na área produtiva; (c) acompanhamento das reuniões diárias de produção para observação dos comportamentos das lideranças e liderados; e (d) realizar visitas as áreas de suporte, tais como administrativas (compras e comercial) e de P\&D (projeto e desenvolvimento) para um maior entendimento do fluxo de atividades e informações que influenciam o processo.

A etapa $(v)$ trata os dados coletados, consolidando as ideias e levando em consideração o contexto em que estas foram colocadas, além de analisar a extensão dos comentários e a especificidade das respostas (TORTORELLA et al., 2008). Assim, define-se o portfólio de práticas enxutas, barreiras e FCS pertinentes ao contexto da pequena empresa em estudo. Além disso, esta etapa permite agrupar informações que são utilizadas para explicar ou justificar as intensidades de relacionamento entre estes elementos, confrontando com a realidade encontrada.

A etapa ( $v i)$ visa analisar as relações entre a implementação das práticas enxutas e as barreiras e FCS, elencados em ( $v$ ). Para tanto, faz-se uso de uma ferramenta de suporte à decisão multicritério denominada AHP (Analytic Hierarchy Process ou Processo Hierárquico Analítico) (SAATY, 1980). O AHP permite a identificação da melhor alternativa em um grupo de variáveis tendo em vista critérios pré-definidos de decisão (TORTORELLA; FOGLIATTO, 2008). Optou-se pela adoção do AHP por ser um processo de decisão estruturado e documentado, apresentando a possibilidade de repetição e aplicação a situações que envolvem julgamentos subjetivos. Assim, este utiliza tanto dados quantitativos quanto qualitativos para definir os valores das preferências (STEIGUER et al., 2003). Está técnica é composta pelas fases: (a) organização do problema em um nível Revista Produção Online. Florianópolis, SC, v. 18, n. 4, p. 1422-1444, 2018. 
hierárquico que reflita as relações existentes entre os critérios de decisão e as variáveis; (b) comparação pareada entre elementos posicionados em um nível hierárquico com relação a elementos no nível superior adjacente; e (c) análise das matrizes de comparações pareadas, geradas através do cálculo de autovetores e autovalores (VARGAS, 2010).

Assim, a Figura 2 apresenta uma hierarquia contendo três níveis. No nível mais alto, identifica-se o objetivo principal da análise multicritério, que é determinar os FCS e barreiras mais relevantes para possibilitar uma implementação bem sucedida da PE em pequenas empresas. No segundo nível, apresenta-se as práticas $p_{x}(x=1, \ldots, 19)$ e no terceiro nível os fatores críticos de sucesso $f_{y}(y=1, \ldots, 13)$ e as barreiras $b_{z}(z=1, \ldots, 4)$, ambos previamente elencados na etapa $(v)$. Para a análise desta hierarquia são necessárias $(x+1)$ matrizes de comparação pareadas. $A$ primeira matriz, relaciona os níveis 1 e 2 da hierarquia. Trata-se de uma matriz onde as comparações pareadas são avaliadas utilizando opinião de especialistas. Os especialistas devem ser escolhidos de acordo com a sua experiência tanto acadêmica quanto prática em relação a implementação de PE. Para tanto realiza-se entrevistas semi-estruturadas com duração planejada de 1 hora, a fim de avaliar as relações entre os níveis. Então, cada par de itens $(i, j)$ é avaliado em importância utilizando uma escala discreta de 1 a 9 , onde 1 denota um cenário em que os itens $i$ e j são igualmente importantes, e 9 denota um cenário em que o item $i$ é extremamente mais importante do que o item $j$. Valores intermediários denotam situações de compromisso e valores recíprocos, tal como (1/9), denotam situações nas quais o item $i$ é menos importante do que o item $j$.

Figura 2 - Níveis de hierarquia da análise multicritério

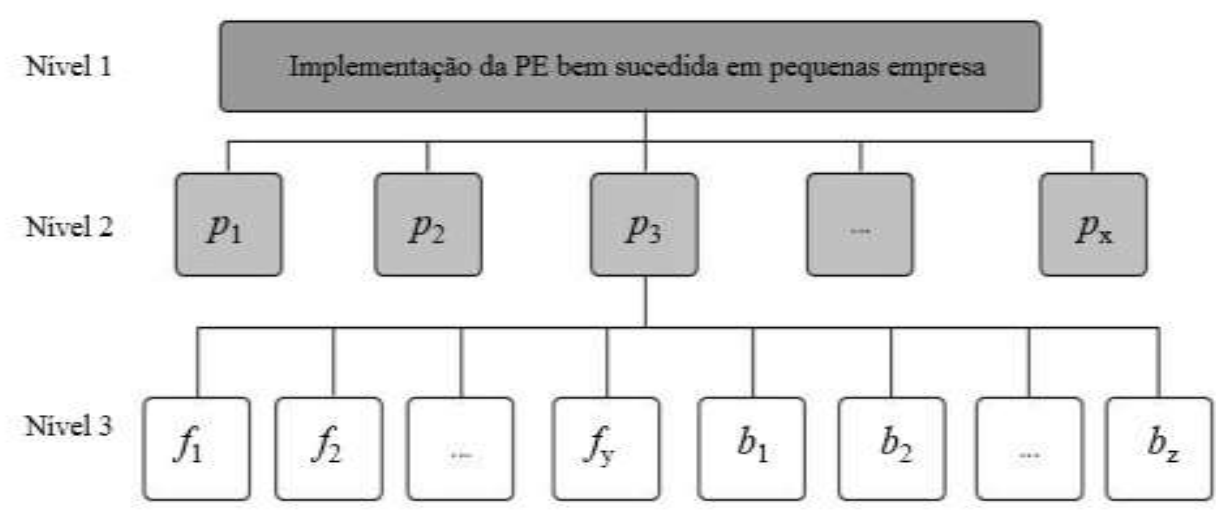


$\mathrm{Na}$ primeira matriz de comparação pareada dos niveis 1 e 2 da hierarquia, tem-se também o vetor de pesos de importância obtido para os elementos listados nas linhas da matriz, definindo, assim, o ranking de importância entre os FCS e barreiras para a implementação da PE. $O$ vetor de pesos de importância corresponde ao autovetor principal, com valores normalizados tal que a soma dos pesos seja igual a 1,0. As demais $x$ matrizes de comparação relacionam os níveis 2 e 3 da hierarquia também definidas através da opinião de especialistas. Cada uma destas matrizes traz as comparações pareadas das nove opções de FCS e barreiras para o processo de implementação em PE com relação a cada prática do nível 2. Em todas as matrizes de comparação pareada os valores para os relacionamentos ij foram obtidos a partir das medianas das respostas dos especialistas, de modo a evitar a consideração de valores espúrios, segundo recomendado por Tortorella e Fogliatto (2014).

Similar ao procedimento utilizado para definição da importância dos critérios de avaliação, são obtidos os vetores de pesos para cada critério de acordo com as opções de FCS e barreiras. Os valores de CR (Razão de Consistência) são gerados com base na consistência das avaliações qualitativas realizadas nos níveis 2 e 3 da hierarquia. Saaty (1980) propõe um valor máximo de 0,10 de CR para que avaliações em uma matriz de comparações sejam consideradas consistentes.

Para maiores detalhes sobre escalas, cálculo de vetor de pesos e determinação da consistência das avaliações, recomendam-se os trabalhos de Saaty e Vargas (1987) e Winston (1997). Estando de acordo os valores identificados, conclui-se que as avaliações feitas pelos especialistas são consistentes, podendo ser utilizadas no restante do método. Para obter o vetor final de pesos, configurando a etapa final de (vii) ranqueamento dos FCS e barreiras para favorecer a implementação enxuta em pequenas empresas, calcula-se a média ponderada das avaliações obtidas para cada alternativa de implementação da PE. Os elementos ponderadores são os pesos de importância atribuídos a cada critério, de modo que a maior pontuação geral configura as caracteristicas necessárias para o favorecimento da implementação da PE em pequenas empresas. Portanto, com base no método proposto, apresenta-se os resultados obtidos após a realização das sete etapas descritas nesta seção. 


\section{RESULTADOS}

A empresa em estudo é caracterizada por uma sociedade limitada (Ltda) e está localizada na capital de Santa Catarina, sendo o ramo de atuação o setor eletrônico. Fundada em 1990 por 3 sócios, engenheiros eletricistas e mestres em eletrônica de potência, a empresa possui experiência no projeto, desenvolvimento e fabricação fontes de alimentação chaveada (conversores CA/CC, conversores $\mathrm{CC} / \mathrm{CC}$, inversores CC/CA, carregadores de baterias, fonte USB, entre outros). Seus clientes são de diversos segmentos tais como bancário, automotivo, telecomunicação, agrícola, etc. A empresa apresenta uma equipe estratégica composta por gerentes das áreas de Produção, P\&D, Administrativo-financeiro, Suprimentos, Comercial e RH (recursos humanos), além dos 3 sócios-diretores. Todas as áreas procuram se comunicar e interagir para resolução de problemas, porém a comunicação entre as áreas de produção, suprimentos e projetos (P\&D) deve fluir de maneira que não haja grandes falhas no processo. O processo produtivo desta empresa apresenta uma equipe com 34 funcionários e 4 líderes de setor que englobam todo o processo de montagem dos conversores (Montagem SMD, Montagem de Magnéticos, Montagem PTH, Teste e Montagem Mecânica/Embalagem), com uma demanda média de 6 mil fontes por mês distribuídas em 20 modelos de produtos, sendo, portanto, classificada como uma pequena empresa tanto pelo faturamento anual como pelo número de funcionários.

O controle de todo o processo produtivo é realizado através de entradas de tempo diárias, de maneira que os operadores estão vinculados às ordens de fabricação (OFs) em tempo real. Isto pode ser verificado pelo sistema integrado, no qual os tempos médios de fabricação e realização permitem a rastreabilidade de todo o processo produtivo. A empresa apresenta iniciativas pontuais em se tratando de PE havendo o auxílio externo de consultores, há mais de 10 anos, com foco no chão de fábrica. Atualmente está implementação tem sido guiada de forma isolada sem integração de todas as áreas envolvidas no processo, tais como Planejamento e controle da produção (PCP), Compras, P\&D, etc.

Quanto à realização dos grupos focados, dois grupos diferentes foram formados. Para a composição do grupo 1 optou-se por convidar membros da equipe estratégica da empresa composto por: um diretor, o gerente de produção, a gerente de suprimentos e o gerente de P\&D. Todos os membros deste grupo apresentam Revista Produção Online. Florianópolis, SC, v. 18, n. 4, p. 1422-1444, 2018. 
experiência mínima de 2 anos na empresa e formação acadêmica de nível superior. O grupo 2 é composto por 4 líderes dos setores produtivos, sendo que todos possuem ensino médio completo e experiência mínima de 2 anos no cargo de liderança.

As reuniões com os grupos focados foram realizadas na sede da empresa, com agendamento e pauta previamente ajustada. Foi prevista uma duração de 60 minutos para cada reunião, sendo que a reunião com o grupo 1 atingiu o tempo planejado de uma hora e a reunião com o grupo 2 durou em torno de 70 minutos. Foram tomadas medidas para evitar interrupções e desatenções dos participantes. Apesar de uma das reuniões ter se estendido um pouco mais que o previsto, não houve evidência de cansaço ou impaciência por parte dos membros do grupo, indicando um bom aproveitamento do tempo para coleta de informações. Além disso, não houve problemas de pontualidade para iniciar ambos os encontros e todos aceitaram que os encontros fossem gravados para registro dos dados coletados. Os membros de ambas os encontros foram cooperativos e participantes, questionando quando não havia o entendimento de determinada prática enxuta, gerando uma expectativa positiva, de interesse e um clima amistoso durante os trabalhos. Houve opiniões dissidentes ao longo do trabalho, que foram adequadamente exploradas pela moderadora, sem que se tenha observado alterações no clima do trabalho. Estas divergências reforçam a hipótese de diversidade observada nos setores ou áreas da empresa. Assim, julga-se que os participantes tenham compreendido as questões e os seus objetivos, tendo emitido respostas e opiniões transparentes, honestas e sinceras.

Para a etapa de observação participativa, o pesquisador teve a oportunidade de vivenciar a rotina da empresa em um período de quinze dias, apresentando uma rotina constante de observações diárias para melhor entendimento das etapas produtivas do processo. Durante este período, o pesquisador observou a gerente de produção na geração das OFs e acompanhamento das mesmas no chão de fábrica, podendo entender melhor as limitações de processo/operação da pequena empresa. Houve também o acompanhamento das reuniões diárias de produção, nas quais pode-se identificar os vários tipos de lideranças existentes e a maneira com a qual elas se comunicam. A partir destas reuniões, observou-se que por ser uma pequena empresa a facilidade de validar as informações do sistema diretamente no chão de fábrica já faz parte da rotina diária de suas lideranças. Por uma questão de espaço Revista Produção Online. Florianópolis, SC, v. 18, n. 4, p. 1422-1444, 2018. 
físico, notou-se a existência de uma certa distância entre as áreas produtivas e as áreas de suporte; porém, as áreas de suporte interagem de maneira rápida por estarem em um mesmo espaço, favorecendo a comunicação verbal entre estas e via sistema interno ou telefone entre a produção e as demais áreas.

Após a análise dos áudios dos dois encontros com grupos focados e das anotações realizadas pela observação participativa, identificou-se que ambos os grupos acreditam que a empresa no longo prazo deverá crescer utilizando de tecnologias mais avançadas e com uma estrutura tanto física quanto organizacional maior. A importância da melhoria continua é evidente e indispensável para que esse crescimento almejado seja alcançado. Assim, para a seleção das práticas potencialmente aplicáveis houve o consenso na eliminação de alguns itens da lista original, seja por motivos de difícil aplicabilidade ou pela falta de maturidade do processo atual em relação aos princípios enxutos. Assim, das 19 práticas enxutas apresentadas apenas 9 foram definidas como aplicáveis na pequena empresa em estudo. Em relação aos FCS e barreiras, o mesmo processo ocorreu de maneira consensual analisando as características da empresa, e 7 FCS e 2 barreiras foram identificados como relevantes de uma lista original de 17 itens. A Tabela 3 consolida as práticas enxutas, barreiras e FCS selecionados a partir dos grupos focados.

Tabela 3 - Práticas, FCS e barreiras elencados para a pequenas empresas

\begin{tabular}{|c|c|}
\hline Práticas enxutas & FCS/Barreiras \\
\hline$p_{1}$ - Produção puxada & $f_{1}-$ Liderança \\
\hline$p_{2}-$ Manutenção produtiva total & $f_{2}-$ Cultura \\
\hline$p_{3}$ - Kaizen / Grupos de melhoria continua & $f_{4}$ - Alinhamento à estratégia em todos os níveis \\
\hline$p_{4}-5 S$ & $f_{5}$ - Educação e treinamento \\
\hline$p_{11}$ - Mapeamento de Fluxo de Valor (MFV) & $\begin{array}{l}f_{7} \text { - Comunicação (metas e objetivos com iniciativas de } \\
\text { melhoria) }\end{array}$ \\
\hline$p_{12}$ - Padronização do trabalho & $f_{10}$ - Aprendizagem contínua \\
\hline$p_{14}$ - Equipe multifuncional & $f_{11}$ - Comprometimento/ Motivação dos trabalhadores \\
\hline$p_{16}-$ Gerenciamento visual & $b_{1}$ - Falta de compreensão dos benefícios \\
\hline$p_{19}$ - Plan, do, check, act (PDCA) & $b_{2}$ - Resistência à mudança pelos funcionários \\
\hline
\end{tabular}

Com base nas duas listas apresentadas na Tabela 3 realizou-se a análise multicriterial cuja a hierarquia está apresentada na Figura 2. Nesse sentido, 10 matrizes de comparação pareada foram analisadas pelos especialistas, cujos valores medianos foram inseridos. Todos os especialistas envolvidos possuem doutorado em sistemas de produção enxuta, com pelo menos 5 anos de experiência 
prática e acadêmica. A Tabela 4 apresenta na primeira linha os pesos das práticas relacionadas para a matriz de comparação dos níveis 1 e 2 , as demais colunas revelam os pesos de comparação dos níveis 2 e 3 , e a coluna à direita revela 0 ranqueamento final para análise. As 10 matrizes de comparação pareada apresentaram valores de CR dentro do esperado confirmando a consistência das avaliações.

A partir dos pesos das práticas, foi identificado que a prática p16 (Gerenciamento Visual) apresenta maior importância para implementação da PE em pequenas empresas. Curiosamente, este resultado diverge de estudos anteriores, visto que a frequência de citação desta prática na literatura é relativamente menor que as demais (ver Tabela 2). Durante as entrevistas com os especialistas, alguns comentários emergiram como forma de justificar tal avaliação. Dentre estes, cabe destacar o fato de tal prática estar intrinsicamente associada com a implementação de várias outras, tais como produção puxada, 5S e padronização do trabalho. Assim, entende-se que esta prática não só apresenta benefícios específicos de sua implementação, mas também fornece subsídio para a implementação das demais, funcionando como uma etapa elementar (pré-requisito) dessas outras. Quanto ao cenário da empresa em estudo, com base nas observações realizadas, percebe-se que esta prática foi implementada de maneira superficial sendo evidenciada apenas através da identificação dos produtos ao longo do processo. Assim, o gerenciamento visual apresenta grande potencial de adoção na empresa, integrando as células produtivas de maneira a ordenar visualmente as prioridades dentro do processo e permitindo que a ocorrência de anomalias se torne de fácil identificação e, consequentemente, rápida tratativa. De um modo geral, as pequenas empresas tendem a buscar práticas enxutas cuja o custo de implementação seja baixo e de rápidos resultados (DORA et al., 2014; ZHOU, 2016). Logo, este resultado corrobora com tal necessidade, uma vez que o gerenciamento visual é uma prática simples e de baixo aporte de capital.

Por outro lado, a prática $p_{11}$ (MFV) possui menor peso para a implementação enxuta bem-sucedida em pequenas empresas. Em termos teóricos, esta prática apresenta uma incidência ligeiramente maior ( 7 em 26 referências pesquisadas) que a p16. Além disso, as justificativas apontadas pelos especialistas entrevistados para esta avaliação contemplam o fato de que pequenas empresas, de um modo geral, apresentam fluxos de valor de menor complexidade nos quais a identificação dos Revista Produção Online. Florianópolis, SC, v. 18, n. 4, p. 1422-1444, 2018. 
desperdícios ocorre de modo mais fácil e notório. Assim, a utilização de práticas que favoreçam um entendimento sistêmico e identificação de perdas de processos mais amplos, tal como o MFV, têm seu potencial de contribuição reduzido dada a simplicidade dos fluxos de valor em questão. No caso da empresa em estudo, observa-se que tanto a gerência quanto a operação apresentam pouca familiaridade com tal prática, direcionando, assim, as iniciativas de melhoria com base em necessidades latentes de curto prazo. Tal fato também é justificado pela multifuncionalidade intrínseca ao contexto das pequenas empresas. Devido baixa disponibilidade de recursos humanos, seus líderes desempenham diversos papéis integrando funções que, em um cenário de grandes empresas, compreenderiam mais de um departamento. Assim, os líderes de pequenas empresas são encorajados a terem uma visão muito mais horizontal dos processos que gerenciam (visão menos departamentalizada), possibilitando maior compreensão do fluxo de valor como um todo e reduzindo a necessidade de práticas que auxiliem tal visão, como o MFV.

Tabela 4 - Matriz com os vetores pesos para as práticas e FCS/barreiras

\begin{tabular}{|c|c|c|c|c|c|c|c|c|c|c|}
\hline & $p_{1}$ & $p_{2}$ & $p_{3}$ & $p_{4}$ & $p_{11}$ & $p_{12}$ & $p_{14}$ & $p_{16}$ & $p_{19}$ & Pesos finais dos \\
\hline $\begin{array}{l}\text { Pesos das } \\
\text { práticas }\end{array}$ & 0,145 & 0,078 & 0,108 & 0,136 & 0,038 & 0,158 & 0,064 & 0,183 & 0,091 & FCS/barreiras \\
\hline$f_{1}$ & 0,10 & 0,12 & 0,14 & 0,06 & 0,13 & 0,07 & 0,10 & 0,09 & 0,12 & 0,096 \\
\hline$f_{2}$ & 0,17 & 0,17 & 0,17 & 0,17 & 0,07 & 0,15 & 0,08 & 0,14 & 0,09 & 0,145 \\
\hline$f_{4}$ & 0,06 & 0,05 & 0,04 & 0,02 & 0,13 & 0,04 & 0,04 & 0,05 & 0,10 & 0,052 \\
\hline$f_{5}$ & 0,13 & 0,18 & 0,09 & 0,17 & 0,16 & 0,16 & 0,23 & 0,12 & 0,19 & 0,150 \\
\hline$f_{7}$ & 0,05 & 0,06 & 0,05 & 0,04 & 0,06 & 0,05 & 0,11 & 0,19 & 0,04 & 0,078 \\
\hline$f_{10}$ & 0,08 & 0,12 & 0,07 & 0,06 & 0,21 & 0,09 & 0,21 & 0,07 & 0,13 & 0,096 \\
\hline$f_{11}$ & 0,14 & 0,10 & 0,23 & 0,22 & 0,05 & 0,18 & 0,12 & 0,13 & 0,08 & 0,152 \\
\hline$b_{1}$ & 0,08 & 0,05 & 0,05 & 0,12 & 0,14 & 0,09 & 0,04 & 0,14 & 0,15 & 0,100 \\
\hline$b_{2}$ & 0,19 & 0,15 & 0,16 & 0,13 & 0,05 & 0,17 & 0,06 & 0,08 & 0,11 & 0,131 \\
\hline CR & 0,03 & 0,03 & 0,04 & 0,01 & 0,01 & 0,02 & 0,04 & 0,09 & 0,02 & \\
\hline
\end{tabular}

Quantos aos FCS/barreiras avaliados, os fatores o $f_{11}$ (Comprometimento/ Motivação dos trabalhadores) e $f_{5}$ (Educação e treinamento) foram os que apresentaram maior peso para a implementação enxuta, com valores de 0,152 e 0,150 respectivamente. Na literatura investigada (vide Tabela 1 ), ambos os fatores apresentam valores intermediários de frequência de citação (11 e 8 citações dentre 
26 referências, respectivamente), sem nenhum destaque em especial. Contudo, de acordo com 0 entendimento dos especialistas, $f_{11}$ é 0 fator mais relevante para implementação de 3 ( $p_{3}, p_{4}$ e $p_{12}$ ) das 9 práticas enxutas elencadas pelas lideranças da pequena empresa. Além disso, dado que a $p_{12}$ (Padronização do trabalho) tem um alto peso $(0,158)$ dentre as práticas enxutas, a relevância de $f_{11}$ aumenta ainda mais. A implementação enxuta, segundo Tortorella et al. (2015), é composta tanto por aspectos técnicos quanto socioculturais. Assim, além do conhecimento tangível, tal implementação requer mudanças de ordem comportamental de modo a sustentar os aspectos técnicos no longo prazo. Dessa forma, a alta importância do comprometimento e motivação dos trabalhadores é consistente com os indicativos de Bhasin (2012b) e Manville et al. (2012). Além disso, em termos práticos, pequenas empresas tendem a apresentar poucos níveis hierárquicos, acarretando em maior grau de autonomia de seus funcionários. Tal fato enaltece a importância de altos níveis de comprometimento dos trabalhadores, em especial, com o processo de implementação enxuta. A justificativa para o alto valor de importância de $f_{5}$ pode ser encontrada nos estudos realizados por Achanga et al. (2006) e Antony et al. (2005), os quais afirmam que as inciativas de capacitação dos funcionários relativas às práticas e princípios da $P E$ são essencialmente mais escassas em pequenas empresas do que em grandes empresas. Assim, a intensificação de atividades de educação e treinamento em prol da implementação enxuta em pequenas empresas está alinhada com 0 entendimento dos especialistas entrevistados, em especial para as práticas $p_{2}$, $p_{14}$ e $p_{19}$.

Em contrapartida, $f_{4}$ (Alinhamento à estratégia em todos os níveis) apresentou menor valor de importância $(0,052)$, sendo o fator de menor importância em 6 ( $p_{2}, p_{3}$, $p_{4}, p_{12}, p_{14}$ e $\left.p_{16}\right)$ das 9 práticas analisadas. Apesar de $f_{4}$ ser citado em 10 das 26 referências apontadas na Tabela 1, os especialistas indicam que, aos poucos níveis hierárquicos compreendidos em pequenas empresas, a comunicação e alinhamento dos objetivos estratégicos tende a ser realizado de maneira mais direta e fluida. Assim, fatores que suportem o desenvolvimento destas atividades têm pouco potencial de contribuição dado o contexto das pequenas empresas. Isto pode ser evidenciado a partir das visitas à empresa em estudo, cujas lideranças de nível estratégico (gerência e diretoria) e tático/operacional (líderes de produção) totalizam uma equipe de 9 pessoas que se encontram no mesmo ambiente de trabalho com reuniões frequentes (uma vez ao dia) e contato informal facilitado. Portanto, o Revista Produção Online. Florianópolis, SC, v. 18, n. 4, p. 1422-1444, 2018. 
resultado encontrado para este fator corrobora com a realidade observada na empresa em estudo.

\section{CONCLUSÕES}

O presente trabalho apresentou um método para identificar o relacionamento entre os fatores críticos de sucesso, barreiras e práticas para a implementação enxuta em uma pequena empresa. Duas grandes contribuições podem ser destacadas neste estudo. Primeiramente, em termos teóricos, a proposição de um método que avalie de forma integrada FCS, barreiras e práticas para a implementação da PE em pequenas empresas contribui para o corpo de conhecimento na área. Estudos anteriores abordam tais aspectos de maneira isolada, sem correlacioná-los ao contexto das pequenas empresas. Assim, a integração de uma ferramenta de análise multicriterial a métodos qualitativos de pesquisa (grupos focados, entrevistas semiestruturadas e observação participativa) permitiu quantificar estes relacionamentos de modo a identificar de esforços para a implementação enxuta.

Segundo, em termos práticos, a obtenção de um ranqueamento dos FCS e barreiras para a implementação enxuta em uma pequena empresa possibilita aos gestores tomadas de decisão que antecipem dificuldades futuras. De acordo com Marodin e Saurin (2015), os FCS e barreiras da implementação enxuta não são de fácil gestão e carecem de tempo para o seu redirecionamento por parte da gerência. Assim, a identificação prévia dos FCS e barreiras que mais afetam o processo de implementação enxuta agiliza as mudanças de ordem técnica e sociocultural inerentes à PE. Além disso, estas diretrizes viabilizam a priorização de esforços gerenciais que tragam maiores benefícios à implementação enxuta no contexto da empresa em questão.

Quanto as limitações deste estudo, cabe destacar que os resultados aqui obtidos não podem ser totalmente generalizáveis às pequenas empresas que visam a implementação da PE. Como as práticas, barreiras e FCS sofreram uma crítica inicial de acordo com a realidade atual da empresa em estudo, a seleção destes pode ser alterada caso outras empresas apresentem diferentes contextos. Além disso, os relacionamentos entre os aspectos supracitados foram analisados sob uma perspectiva linear, desconsiderando o caráter sistêmica da implementação enxuta. Em outras palavras, estudos futuros que abordem estes relacionamentos sob a perspectiva de sistemas complexos podem identificar efeitos complementares ou até mesmo divergentes dos aqui relatados. Para tal, sugere-se a incorporação de técnicas voltadas à dinâmica de sistemas para continuidade destes estudos. 


\section{REFERÊNCIAS}

ABOLHASSANI, A.; LAYFIELD, K.; GOPALAKRISHNAN, B. Lean and US manufacturing industry: popularity of practices and implementation barriers. International Journal of Productivity and Performance Management, v. 65, n. 7, p. 875-897, 2016. DOI: https://doi.org/10.1108/IJPPM-10-2014-0157

ACHANGA, P. SHEHAB, E.; ROY, R.; NELDER, G. Critical success factors for lean implementation within SMEs. Journal of Manufacturing Technology Management, v. 17, n. 4, p. 460-471, 2006. DOI: https://doi.org/10.1108/17410380610662889

ANAND, G.; KODALI, R. Analysis of lean manufacturing frameworks. Journal of Advanced Manufacturing Systems, v. 9, n. 01, p. 1-30, 2010. DOI:

https://doi.org/10.1142/S0219686710001776

ANTONY, J., KUMAR, M. \& MADU, C.N. (2005), Six sigma in small- and medium-sized UK manufacturing enterprises: Some empirical observations, The International Journal of Quality \& Reliability Management, Vol. 22, No. 8/9, pp. 860-874. DOI:

https://doi.org/10.1108/02656710510617265

ANTONY, J.; KUMAR, M.; MADU, C. N. Six sigma in small-and medium-sized UK manufacturing enterprises: Some empirical observations. International Journal of Quality \& Reliability Management, v. 22, n. 8, p. 860-874, 2005. DOI: https://doi.org/10.1108/02656710510617265

BAKAS, O.; GOVAERT, T.; VAN LANDEGHEM, H. Challenges and success factors for implementation of lean manufacturing in European SMES. In: 13th International conference on the Modern Information Technology in the Innovation Processes of the Industrial Enterprise (MITIP 2011). Tapir Academic Press, 2011.

BELHADI, A.; TOURIKI, F. A framework for effective implementation of lean production in Small and Medium-sized Enterprises. Journal of Industrial Engineering and Management, v. 9, n. 3, p. 786-810, 2016. DOI: https://doi.org/10.3926/iiem.1907

BEYEA, S., NICOLL, L.H. Methods to conduct focus group and the moderator's rule. Association Of Operating Room Nurses Journal, Denver, v.71, n.5, p.1067-1068, 2000. DOI: https://doi.org/10.1016/S0001-2092(06)61558-5

BHAMU, J.; SANGWAN, K. S. Lean manufacturing: literature review and research issues. International Journal of Operations \& Production Management, v. 34, n. 7, p. 876-940, 2014. DOi: https://doi.org/10.1108/IJOPM-08-2012-0315

BHASIN, S. Prominent obstacles to lean. International Journal of Productivity and Performance Management, v. 61, n. 4, p. 403-425, 2012. DOI: https://doi.org/10.1108/17410401211212661

DOMBROWSKI, U.; CRESPO, I.; ZAHN, T. Adaptive configuration of a lean production system in small and medium-sized enterprises. Production Engineering, v. 4, n. 4, p. 341348, 2010. DOI: https://doi.org/10.1007/s11740-010-0250-5

DOMBROWSKI, U.; MIELKE, T. Lean leadership-15 rules for a sustainable lean implementation. Procedia CIRP, v. 17, p. 565-570, 2014. DOI:

https://doi.org/10.1016/i.procir.2014.01.146 
DOOLEN, T. L.; HACKER, M. E. A review of lean assessment in organizations: an exploratory study of lean practices by electronics manufacturers. Journal of Manufacturing systems, v. 24, n. 1, p. 55-67, 2005. DOI: https://doi.org/10.1016/S0278-6125(05)80007-X

DORA, M.; VAN GOUBERGEN, D.; KUMAR, M.; MOLNAR, A.; GELLYNCK, X. Application of lean practices in small and medium-sized food enterprises. British Food Journal, v. 116, n. 1, p. 125-141, 2014. DOI: https://doi.org/10.1108/BFJ-05-2012-0107

DORA, M.; KUMAR, M.; VAN GOUBERGEN, D.; MOLNAR, A.; GELLYNCK, X. Operational performance and critical success factors of lean manufacturing in European food processing SMEs. Trends in Food Science \& Technology, v. 31, n. 2, p. 156-164, 2013. DOI: https://doi.org/10.1016/j.tifs.2013.03.002

GODINHO FILHO, M.; GANGA, G. M.; GUNASEKARAN, A. Lean manufacturing in Brazilian small and medium enterprises: implementation and effect on performance. International Journal of Production Research, v. 54, n. 24, p. 7523-7545, 2016. DOI:

https://doi.org/10.1080/00207543.2016.1201606

HALLGREN, M.; OLHAGER, J. Lean and agile manufacturing: external and internal drivers and performance outcomes. International Journal of Operations \& Production Management, v. 29, n. 10, p. 976-999, 2009. DOI: https://doi.org/10.1108/01443570910993456

KUMAR, M.; ANTONY, J. Multiple case-study analysis of quality management practices within UK Six Sigma and non-Six Sigma manufacturing small-and medium-sized enterprises. Proceedings of the Institution of Mechanical Engineers, Part B: Journal of Engineering Manufacture, v. 223, n. 7, p. 925-934, 2009. DOI:

https://doi.org/10.1243/09544054JEM1288

KUMAR, M.; ANTONY, J.; DOUGLAS, A.. Does size matter for Six Sigma implementation? Findings from the survey in UK SMEs. The TQM journal, v. 21, n. 6, p. 623-635, 2009. DOI: https://doi.org/10.1108/17542730910995882

KUMAR, M.; KHURSHID, K. K.; WADDELL, D. Status of quality management practices in manufacturing SMEs: a comparative study between Australia and the UK. International Journal of Production Research, v. 52, n. 21, p. 6482-6495, 2014. DOI: https://doi.org/10.1080/00207543.2014.948574

KUMAR, M.; ANTONY, J.; SINGH, R.; TIWARI, M.; PERRY, D. Implementing the Lean Sigma framework in an Indian SME: a case study. Production Planning and Control, v. 17, n. 4, p. 407-423, 2006. DOI: https://doi.org/10.1080/09537280500483350

MANVILLE, G.; GREATBANKS, R.; KRISHNASAMY, R.; PARKER, D. W. Critical success factors for Lean Six Sigma programmes: a view from middle management. International Journal of Quality \& Reliability Management, v. 29, n. 1, p. 7-20, 2012. DOI: https://doi.org/10.1108/02656711211190846

MARODIN, G. A.; SAURIN, T. A. Managing barriers to lean production implementation: context matters. International Journal of Production Research, v. 53, n. 13, p. 3947-3962, 2015. DOI: https://doi.org/10.1080/00207543.2014.980454

MATT, D. T. Template based production system design. Journal of Manufacturing Technology Management, v. 19, n.7, p. 783-797, 2008. DOI: https://doi.org/10.1108/17410380810898741 
MATT, D. T.; RAUCH, E. Implementation of lean production in small sized enterprises. Procedia CIRP, v. 12, p. 420-425, 2013. DOI:

https://doi.org/10.1016/j.procir.2013.09.072

NETLAND, T. H. Critical success factors for implementing lean production: the effect of contingencies. International Journal of Production Research, v. 54, n. 8, p. 2433-2448, 2016. DOI: https://doi.org/10.1080/00207543.2015.1096976

NOGUEIRA, M. O.; OLIVEIRA, J. M. Da baleia ao ornitorrinco: contribuições para a compreensão do universo das micro e pequenas empresas brasileiras. Instituto de Pesquisa Econômica Aplicada (Ipea), 2013.

NORDIN, N; DEROS, B. Md; WAHAB, D. A. A survey on lean manufacturing implementation in Malaysian automotive industry. International Journal of Innovation, Management and Technology, v. 1, n. 4, p. 374, 2010.

PEREIRA, L.G.; TORTORELLA, G. L., Implementação de produção enxuta em pequenas empresas de manufatura: uma revisão bibliográfica. In: VI Congresso de Sistemas LEAN. p. 375-390, 2016.

RIBEIRO, J. L. D.; RUPPENTHAL, C. S., Grupos focalizados: revisão e emprego na engenharia de produção. Caderno de Engenharia, PPGEP/UFRGS, Porto Alegre-RS, 2000.

RIBEIRO, J. L. D. Grupos focados: teoria e aplicações. Porto Alegre: FEENG, 2003. RIBEIRO, J.; NEWMANN, C. R. Estudos qualitativos com o apoio de Grupos Focados. XIII Semana de Engenharia de Produção Sul-americana. Gramado, 2012.

ROSE, A. M. N.; DEROS, B. Md.; RAHMAN, M. N. Ab.; NORDIN, N. Lean manufacturing best practices in SMEs. In: Proceedings of the 2011 International Conference on Industrial Engineering and Operations Management. p. 872-877. 2011. DOi: https://doi.org/10.15282/ijame.8.2013.33.0121

ROSE, A. N. M.; DEROS, B. Md; AB RAHMAN, M. N. A study on lean manufacturing implementation in Malaysian automotive component industry. International Journal of Automotive and Mechanical Engineering, v. 8, p. 1467, 2013.

SAATY, T. L. The Analytic Hierarchy Process. New York: McGraw-Hill, 1980.

SAATY, T. L.; VARGAS, L. The Legitimacy of Rank Reversal. Omega, 12, 1987.

SANCHEZ, A. M., PEREZ, M. P. Lean indicators and manufacturing strategies. International Journal of Operations \& Production Management, v. 21, n. 11, p. 1433-1452, 2001. DOI: https://doi.org/10.1108/01443570110407436

SAURIN, T. A.; RIBEIRO, J. L.; MARODIN, G. A. Identificação de oportunidades de pesquisa a partir de um levantamento da implantação da produção enxuta em empresas do Brasil e do exterior. Gestão e produção. São Carlos, SP. Vol. 17, n. 4 (out./dez. 2010), p. 829-841, 2010.

SEBRAE. Participação das micro e pequenas empresas na economia brasileira. 106p. Brasília, 2014. 
SHAH, R.; WARD, P. T. Defining and developing measures of lean production. Journal of operations management, v. 25, n. 4, p. 785-805, 2007. DOI:

https://doi.org/10.1016/j.jom.2007.01.019

SHAH, R.; WARD, P. T. Lean manufacturing: context, practice bundles, and

performance. Journal of operations management, v. 21, n. 2, p. 129-149, 2003. DOI:

https://doi.org/10.1016/S0272-6963(02)00108-0

SIM, K. L.; ROGERS, J. W. Implementing lean production systems: barriers to change. Management research news, v. 32, n. 1, p. 37-49, 2008. DOI:

https://doi.org/10.1108/01409170910922014

STEIGUER, J. E.; DUBERSTEIN, J.; LOPES, V. The analytic hierarchy process as a means for integrated watershed management. In: First interagency conference on research on the watersheds. Agricultural Research Service, US Department of Agriculture, Agricultural Research Service, Benson, Ariz. p. 734-740, 2003.

TIMANS, W.; ANTONY, J.; AHAUS, K.; VAN SOLINGEN, R. Implementation of Lean Six Sigma in small-and medium-sized manufacturing enterprises in the Netherlands. Journal of the Operational Research Society, v. 63, n. 3, p. 339-353, 2012. DOI: https://doi.org/10.1057/jors.2011.47

TORTORELLA, G. L.; MARODIN, G.; FOGLIATTO, F.; MIORANDO, R. Learning organisation and human resources management practices: an exploratory research in medium-sized enterprises undergoing a lean implementation. International Journal of Production Research, v. 53, n. 13, p. 3989-4000, 2015. DOI: https://doi.org/10.1080/00207543.2014.980462

TORTORELLA, G. L.; FOGLIATTO, F. S.; RIBEIRO, J. L. D. Identificação de fatores que afetam a sustentabilidade de melhorias em células de manufatura usando grupos focados. XXVIII Encontro Nacional de Engenharia de Produção, 2008. DOI: https://doi.org/10.1080/00207543.2014.881577

TORTORELLA, G. L.; FOGLIATTO, F. S. Method for assessing human resources management practices and organisational learning factors in a company under lean manufacturing implementation. International Journal of Production Research, Vol. 52, No. 15, pp. 4623-4645, 2014. DOI: https://doi.org/10.1590/S0103-65132008000300015

TORTORELLA, G. L.; FOGLIATTO, F. S. Planejamento sistemático de layout com apoio de análise de decisão multicritério. Produção, v. 18, n. 3, p. 609-624, 2008.

VARGAS, R. Utilizando a Programação Multicritério (AHP) para Selecionar e Priorizar Projetos na Gestão de Portfólio. PMI GLOBAL CONGRESS. 2010.

WINSTON, W. L. Operations research: applications and algorithms. 3. ed. New York: Wadsworth, 1997.

WOMACK, J. P.; JONES, D. T. A máquina que mudou o mundo. Rio de Janeiro: Campus, 1992.

WONG, Y. C.; WONG, K. Y.; ALI, A. A study on lean manufacturing implementation in the Malaysian electrical and electronics industry. European Journal of Scientific Research, v. 38, n. 4, p. 521-535, 2009. 
WORLEY, J. M.; DOOLEN, T. L. The role of communication and management support in a lean manufacturing implementation. Management Decision, v. 44, n. 2, p. 228-245, 2006. DOI: https://doi.org/10.1108/00251740610650210

YANG, P. YU, Y. The Barriers to SMEs' Implementation of Lean Production and Counter measures - Based on SMS in Wenzhou. International Journal of Innovation, Management and Technology, v. 1, n. 2, p. 220, 2010.

YEW WONG, K. Critical success factors for implementing knowledge management in small and medium enterprises. Industrial Management \& Data Systems, v. 105, n. 3, p. 261-279, 2005. DOI: https://doi.org/10.1108/02635570510590101

ZHOU, B. Lean principles, practices, and impacts: a study on small and medium-sized enterprises (SMEs). Annals of Operations Research, v. 241, n. 1-2, p. 457-474, 2016. DOI: https://doi.org/10.1007/s10479-012-1177-3

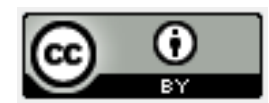

Artigo recebido em: 18/10/2017 e aceito para publicação em: 03/10/2018

DOI: http://dx.doi.org/10.14488/1676-1901.v18i4.3035 